Corresponding authors: ischrader@bccancer.bc.ca; sjones@bcgsc.ca

(C) 2017 Thibodeau et al. This article is distributed under the terms of the Creative Commons Attribution-NonCommercial License, which permits reuse and redistribution, except for commercial purposes, provided that the original author and source are credited.

Ontology terms: neoplasm of the breast; uterine

leiomyosarcoma

Published by Cold Spring Harbor Laboratory Press

doi: 10.1101/mcs.a001628

\section{Genomic profiling of pelvic genital type leiomyosarcoma in a woman with a germline CHEK2:c.1100delC mutation and a concomitant diagnosis of metastatic invasive ductal breast carcinoma}

\author{
My Linh Thibodeau, ${ }^{1}$ Caralyn Reisle, ${ }^{2}$ Eric Zhao, ${ }^{2}$ Lee Ann Martin, ${ }^{3}$ \\ Yazeed Alwelaie, ${ }^{4}$ Karen L. Mungall, ${ }^{2}$ Carolyn Ch'ng, ${ }^{2}$ Ruth Thomas, ${ }^{5}$ Tony Ng, $^{4}$ \\ Stephen Yip, ${ }^{4}$ Howard J. Lim, ${ }^{6}$ Sophie Sun, ${ }^{6}$ Sean S. Young, ${ }^{4,7}$ Aly Karsan, ${ }^{2,4,7}$ \\ Yongjun Zhao, ${ }^{2}$ Andrew J. Mungall, ${ }^{2}$ Richard A. Moore, ${ }^{2}$ Daniel J. Renouf, ${ }^{6}$ \\ Karen Gelmon, ${ }^{6}$ Yussanne P. Ma, ${ }^{2}$ Malcolm Hayes, ${ }^{4,7}$ Janessa Laskin, ${ }^{1,2,9}$ \\ Marco A. Marra, ${ }^{1,2,9}$ Kasmintan A. Schrader, ${ }^{8,9}$ and Steven J. M. Jones ${ }^{1,2,9}$ \\ ${ }^{1}$ Department of Medical Genetics, University of British Columbia, Vancouver, British Columbia V6H 3N1, \\ Canada; ${ }^{2}$ Canada's Michael Smith Genome Sciences Centre, British Columbia Cancer Agency, British \\ Columbia V5Z 4S6, Canada; ${ }^{3}$ Fraser Valley Cancer Centre, British Columbia Cancer Agency, Surrey, British \\ Columbia V3V 1Z2, Canada; ${ }^{4}$ Department of Pathology and Laboratory Medicine, University of British \\ Columbia, Vancouver, British Columbia V5Z 1M9, Canada; ${ }^{5}$ Hereditary Cancer Program, British Columbia \\ Cancer Agency-Abbotsford, Abbotsford, British Columbia V2S 0C2, Canada; ${ }^{6}$ British Columbia Cancer \\ Agency, Vancouver, British Columbia V5Z 4E6, Canada; ${ }^{7}$ Cancer Genetics Laboratory, Department of \\ Pathology and Laboratory Medicine, British Columbia Cancer Agency, Vancouver, British Columbia V5Z 4E6, \\ Canada; ${ }^{8}$ Hereditary Cancer Program, Department of Medical Genetics, British Columbia Cancer Agency, \\ 614-750 West Broadway, Vancouver British Columbia V5Z 1H5, Canada
}

Abstract We describe a woman with the known pathogenic germline variant CHEK2: c.1100delC and synchronous diagnoses of both pelvic genital type leiomyosarcoma (LMS) and metastatic invasive ductal breast carcinoma. CHEK2 (checkpoint kinase 2) is a tumorsuppressor gene encoding a serine/threonine-protein kinase (CHEK2) involved in doublestrand DNA break repair and cell cycle arrest. The CHEK2:c.1100delC variant is a moderate penetrance allele resulting in an approximately twofold increase in breast cancer risk. Whole-genome and whole-transcriptome sequencing were performed on the leiomyosarcoma and matched blood-derived DNA. Despite the presence of several genomic hits within the double-strand DNA damage pathway (CHEK2 germline variant and multiple RAD51B somatic structural variants), tumor profiling did not show an obvious DNA repair deficiency signature. However, even though the LMS displayed clear malignant features, its genomic profiling revealed several characteristics classically associated with leiomyomas including a translocation, $\mathrm{t}(12 ; 14)$, with one breakpoint disrupting RAD51B and the other breakpoint upstream of HMGA2 with very high expression of HMGA2 and PLAG1. This is the first report of LMS genomic profiling in a patient with the germline CHEK2:c.1100delC variant and an additional diagnosis of metastatic invasive ductal breast carcinoma. We also describe a possible mechanistic relationship between leiomyoma and

\footnotetext{
${ }^{9}$ These authors have contributed equally to this work.
} 
COLD SPRING HARBOR Molecular Case Studies
Leiomyosarcoma genomic profiling and CHEK2 variant
LMS based on genomic and transcriptome data. Our findings suggest that RAD51B translocation and HMGA2 overexpression may play an important role in LMS oncogenesis.

[Supplemental material is available for this article.]

\section{INTRODUCTION}

CHEK2 (checkpoint kinase 2) is a tumor-suppressor gene involved in cell cycle arrest and encodes a serine/threonine-protein kinase (CHEK2) activated in response to double-strand DNA damage (Zannini et al. 2014). Multiple case-control studies have reported an association between the truncating variant CHEK2:c.1100delC (NM_007194.3:c.1100delC, rs555607708, hg19 Chr22:g.29091856AG >A) in the heterozygous state and breast cancer risk in BRCA1/BRCA2-mutation-negative women (Meijers-Heijboer et al. 2002; Weischer et al. 2008; Desrichard et al. 2011). The consensus view is that this variant confers an approximately twofold increase in breast cancer risk above that of the general population and is a moderate penetrance allele (Meijers-Heijboer et al. 2002; Weischer et al. 2008; Desrichard et al. 2011). Breast cancers associated with CHEK2:c.1100delC are predominantly estrogen receptor (ER)-positive (Nagel et al. 2012; Weischer et al. 2012; Kriege et al. 2014, 2015). More recently, CHEK2:c.1100delC has been associated with an increased risk of other cancers (hazard ratio [HR] 1.45, 95\% confidence interval [Cl] 1.15-1.82), among which stomach (HR 5.76, 95\% Cl 2.12-15.6) and kidney (HR 3.61, 95\% Cl 1.33-9.79) cancers, as well as sarcomas (HR 3.45, 95\% Cl 1.09-10.9), displayed the highest increase in risk (NäslundKoch et al. 2016). Furthermore, this variant, in its homozygous state, has also been reported in some families with Li-Fraumeni syndrome type 2 (MIM \#609265) (Bell et al. 1999; Vahteristo et al. 2001). No association has been observed with uterine or genital type leiomyosarcoma (LMS), presumably because of the rarity of this tumor type. The incidence rate of LMS in the United States is 12.3 per million (Toro et al. 2006), but it is reported as low as 4 per million in European Nordic countries (Koivisto-Korander et al. 2012). As per the Exome Aggregation Consortium (ExAC) database, the highest minor allele frequency of CHEK2:c.1100delC (NM_007194.3, rs555607708) is in the Finnish population at 0.008, followed by non-Finn Europeans at 0.002 (Lek et al. 2016).

An optimal treatment regime specifically for breast cancer patients carrying the CHEK2: c.1100delC mutation has yet to be elucidated, and it is still unknown why these patients have poorer prognosis when compared with noncarriers or why they are at increased risk of a second metachronous breast cancer (Weischer et al. 2012; Kriege et al. 2014; Pfeifer et al. 2014). A recent study showed that breast cancer risk was similar in CHEK2 founder versus nonfounder mutation carriers and that the risk of multiple primary cancers of different origin was also increased (Leedom et al. 2016).

We identified the CHEK2:c.1100delC variant in the LMS tumor sample as well as the blood from a patient with concomitant diagnoses of both pelvic genital type LMS and metastatic invasive ductal breast carcinoma.

\section{RESULTS}

\section{Clinical Presentation and Family History \\ Patient-Oncological History}

We describe a 61-yr-old female with synchronous diagnoses of pelvic genital type LMS and metastatic breast cancer. 
At $59 \mathrm{yr}$ of age, the patient had abdominal computed tomography (CT) imaging in the context of abdominal pain, which revealed a left nephrolithiasis, multiple uterine fibroids, and what initially appeared to be a right adnexal solid mass $(6.7 \times 7 \mathrm{~cm})$ possibly associated with the uterus. The patient underwent subtotal hysterectomy and bilateral salpingectomyoophorectomy, but the additional separate pelvic mass $(10-12 \mathrm{~cm})$ was firmly adherent to the right posterolateral pelvic wall and could not be excised. Histopathological examination of the uterus showed multiple benign cellular leiomyomata. A repeat pelvic MRI showed a $15-\mathrm{cm}$ centrally necrotic pelvic mass with cystic component and local invasion along the pelvic wall and right sacroiliac joint with mass effect leading to compression of the bladder. Within a 4-mo period, the mass had doubled in size from 7 to $15 \mathrm{~cm}$. A CT-guided transgluteal biopsy of the pelvic mass was obtained and revealed features in keeping with pelvic genital type LMS (Fig. 1). The patient underwent local radiation therapy, subsequent repeat attempted resection (unsuccessful), and then received single-agent adriamycin (six cycles). A CT-guided biopsy of the mass was then obtained at the time of therapeutic pelvic cyst drainage and was used for both genomic and transcriptome profiling (Laskin et al. 2015).

Concurrently, breast imaging (mammography and ultrasound) was performed in the context of a right-sided breast lump and revealed a $7.5 \times 4 \times 3 \mathrm{~cm}$ mass with several abnormal lymph nodes in the right axilla. Fine needle aspiration biopsy showed epithelioid cells suggestive of malignancy and core needle biopsies revealed ER-positive, PR-positive, HER2positive invasive ductal carcinoma (Nottingham grade 2) (Fig. 2). The patient underwent right mastectomy and axillary lymph node dissection.

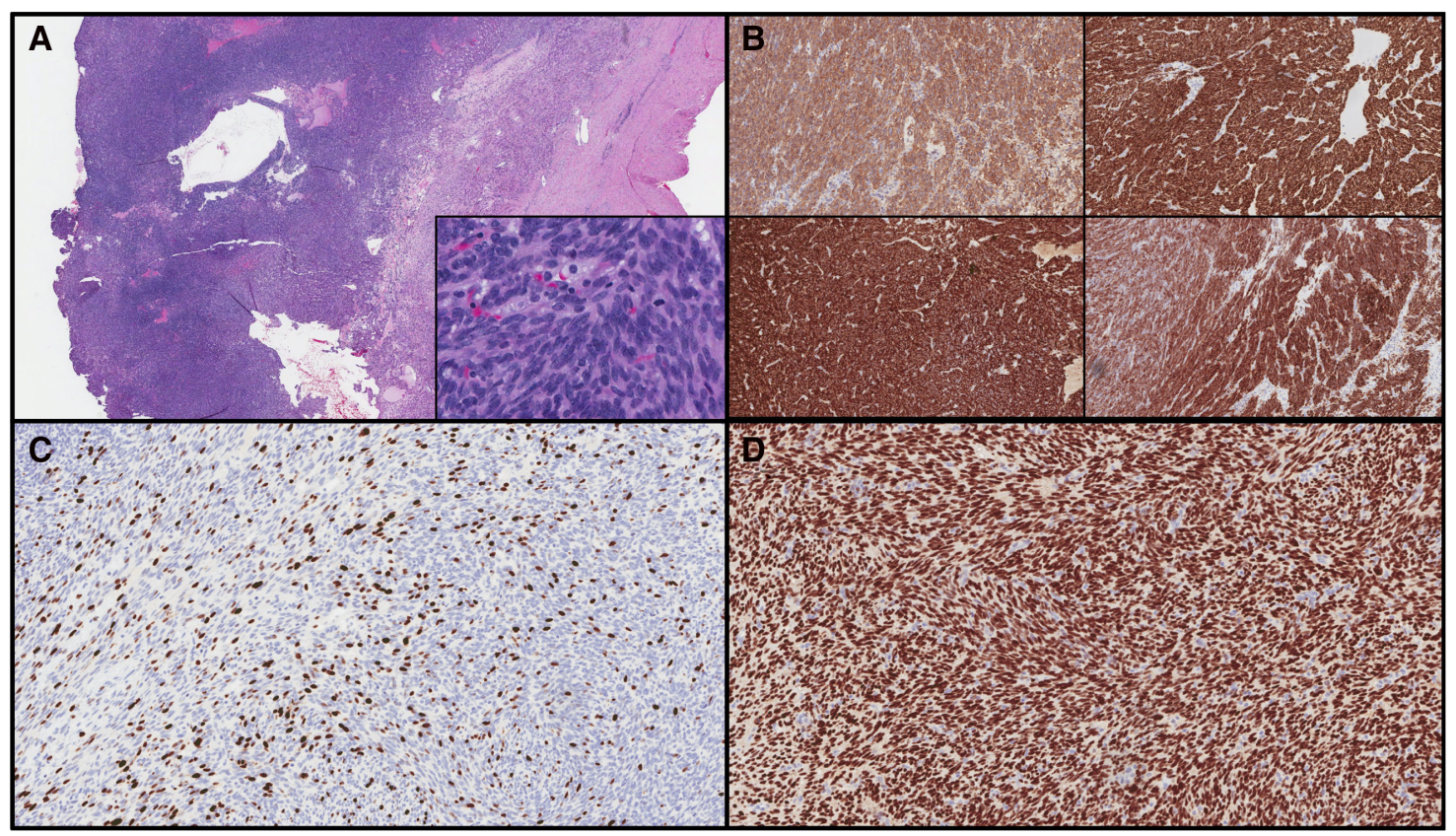

Figure 1. Leiomyosarcoma of genital type. (A) Photomicrograph showing a highly cellular smooth muscle neoplasm with elongated blunt-ended nuclei and abundant eosinophilic cytoplasm characteristic of muscle lineage with hematoxylin and eosin (H\&E) stain (larger image 20× magnification; inset, 200× magnification). (B) Immunostains showing strong and diffuse positive staining for muscle-specific actin (upper left), desmin (upper right), heavy chain myosin (lower left), and $\mathrm{H}$-caldesmon (lower right) (100× magnification). (C) The immunostain for Ki-67 showed a proliferative index of $\sim 20 \%$ in some parts of the tumor (200 $\times$ magnification). (D) The tumor was diffusely positive for estrogen receptors (200× magnification). 


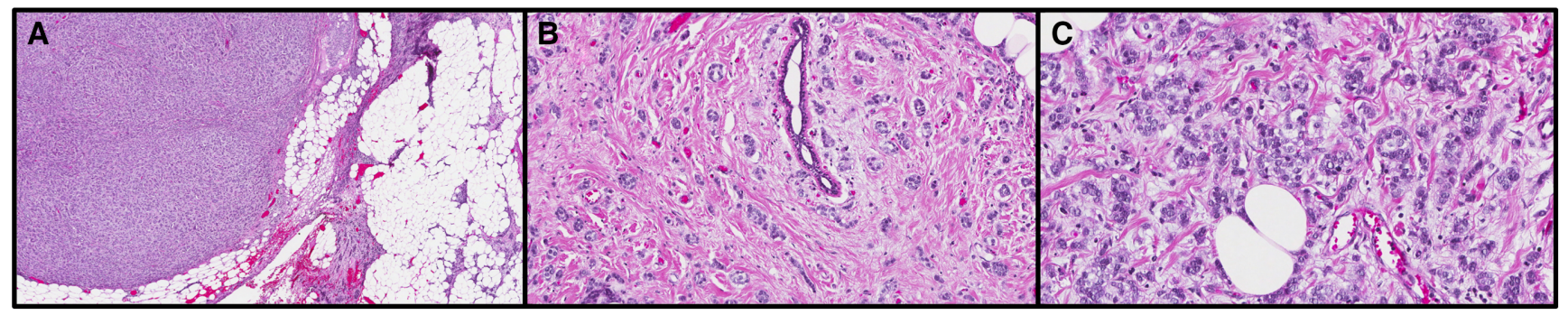

Figure 2. Invasive ductal breast carcinoma with infiltrating margins. (A) Low-power photomicrograph showing invasive ductal carcinoma (H\&E, 40× magnification). (B) Invasive ductal carcinoma surrounding a normal breast duct (H\&E, 100x magnification). (C) Invasive ductal carcinoma displaying grade 2 nuclei (H\&E, 200x magnification).

Initial systemic treatment consisted of letrozole and trastuzumab therapy, whereas radiation and attempted resection was directed toward the unresectable pelvic mass. The patient underwent local radiation therapy with subsequent repeat attempted resection (unsuccessful), and then received single-agent adriamycin (six cycles). Trastuzumab was discontinued after five doses to avoid cardiotoxicity when palliative treatment began with adriamycin/doxorubicin (six cycles) for the unresectable symptomatic LMS.

The patient currently remains on letrozole for treatment of both the pelvic genital type LMS and the postsurgical management of her breast cancer (high risk recurrence). Clinical and radiological assessment is suggestive of pelvic disease stabilization, but subtle signs of slow progression are noted (e.g., adenopathy).

\section{Patient-Other Medical Conditions}

This patient was diagnosed with rheumatoid arthritis in her early 40s. She was treated with rituximab for $8 \mathrm{yr}$, which was discontinued following her breast cancer and pelvic genital type LMS cancer diagnoses. Other comorbidities included insulin resistance, hypothyroidism, hypertension, nephrolithiasis, osteoporosis, and polycystic ovarian syndrome (PCOS).

\section{Family History}

The patient is of Irish (maternal) and mixed Native American/German (paternal) ancestry. She has three healthy daughters. The patient is from a sibship of four. Detailed family history revealed two cases of premenopausal breast cancer in second-degree relatives: a niece, diagnosed with ER-positive breast cancer at age 35 (treated with lumpectomy and radiation therapy) and a paternal aunt, diagnosed with breast cancer in her 30s.

\section{Pathology \\ LMS}

The sections showed a highly cellular smooth muscle neoplasm (Fig. 1A). At the periphery of the lesion, the neoplastic cells were better differentiated and were clearly of smooth muscle lineage having elongated blunt-ended nuclei and abundant eosinophilic cytoplasm. Here, mitotic figures were scanty. Toward the more central region of the tumor the cells were small, had crowded nuclei, and scanty cytoplasm. Zonal necrosis was seen. Mitotic figures numbered up to 10 in 10 high-power fields. Immunostains showed the tumor to be strongly and diffusely positive for muscle-specific actin, desmin, heavy chain myosin, and $\mathrm{H}$-caldesmon (Fig. 1B). The immunostain for Ki-67 showed a proliferative index of $\sim 20 \%$ in the cellular 
areas of the tumor (Fig. 1C). The tumor was diffusely positive for ERs (Fig. 1D). The stain for p16 was positive in $<10 \%$ of tumor nuclei.

Based on the age of the patient, the lack of history of progestogen therapy, the documented increase in size of the mass over the period of a few months, the presence of mitoses, and the presence of necrosis, the tumor was regarded as an LMS of genital type, French grade 2 using the grading system for peripheral soft tissue sarcoma.

\section{Invasive Ductal Carcinoma}

The breast carcinoma was $6 \mathrm{~cm}$ in greatest dimension. Sections showed invasive ductal breast carcinoma (Nottingham grade 2, ER/progesterone receptor (PR)-positive, HER2positive) with infiltrating margins (Fig. 2A,B) and grade 2 nuclei (Fig. 2C). Lymphovascular invasion and metastases to all 18 axillary and intramammary lymph nodes with extensive extranodal extension was evident (pT3, N3a, M0).

\section{Genomic Results}

\section{Germline}

Whole-genome sequencing of a fresh-frozen core biopsy sampling of the LMS (in comparison with whole-genome sequencing of matched peripheral blood) revealed the heterozygous germline CHEK2:c.1100delC truncating variant, present in the germline and retained in the tumor. This was subsequently confirmed clinically via Sanger sequencing of peripheral blood. There was no evidence of a second somatic aberration to the wildtype CHEK2 allele such as loss of heterozygosity (LOH) or a second somatic CHEK2 pathogenic variant. This is in keeping with previous scientific evidence reported in the breast cancer literature (Suspitsin et al. 2014). Furthermore, CHEK2 gene expression was found to be low compared with other sarcomas (SARC) but comparable to normal tissue expression (Table 1).

\section{Somatic}

Briefly, whole-genome sequencing of both LMS and peripheral blood were performed to allow for comparative analysis and detection of somatic single-nucleotide variants (SNVs), indels, structural variants (SVs), and copy-number variants (CNVs). Whole-transcriptome sequencing of the LMS was also performed. The normal transcript comparator used was the compendium average from the Illumina Body Map data set and the disease expression comparator used was predominately the sarcoma (SARC) TCGA data set and to a lesser extent, the average across all TCGA tumor samples. The estimated tumor content was $42 \%$. Please refer to Methods for more details.

The overall mutational burden was unremarkable when compared with the entire TCGA data set with 27 nonsynonymous coding SNVs (30th percentile) and four small indel events (60th percentile). There were 51 structural variants, falling at the 51 st percentile among our local database of 339 diverse cancer cases (Laskin et al. 2015). Please refer to Supplemental Table S1 for a complete list of somatic SNVs, indels, CNVs, and SVs and to Supplemental Figures $\mathrm{S} 1$ and $\mathrm{S} 2$ for the Circos and CNV plots.

Of note, a somatic BRCA1 variant of uncertain significance was also identified (p.Gln1327His) and BRCA1 expression appeared comparable to other tumors (Table 1). The hormone receptor gene expression profile was not an outlier with respect to percentile or fold change (Table 1).

Multiple disruptive structural rearrangements were seen in RAD51B. The first structural variant is a deletion, hg19 Chr14:g.68219215_68986637del (767,422 bp) (Fig. 3A,B), which only leaves the last exon of RAD51B. The second structural variant is a translocation $t(12 ; 14)$ 
Table 1. Copy number RNA expression metrics of selected genes in our leiomyosarcoma (LMS) tumor compared with the gene expression distribution from RNA-seq pooled data of all TCGA tumors, all TCGA matched normal, TCGA sarcoma tumors, and Illumina Human Body Map 2.0 (data from 16 human tissue types)

\begin{tabular}{|c|c|c|c|c|c|c|c|c|}
\hline \multirow[b]{2}{*}{ Gene } & \multirow{2}{*}{$\begin{array}{l}\text { Copy number } \\
\text { (diploid model) }\end{array}$} & \multicolumn{2}{|c|}{ All TCGA } & \multicolumn{2}{|c|}{$\begin{array}{c}\text { All TCGA } \\
\text { (matched } \\
\text { normal) }\end{array}$} & \multicolumn{2}{|c|}{ SARC TCGA } & \multirow{2}{*}{$\frac{\text { Bodymap }}{\text { Mean FC }}$} \\
\hline & & \%tile & $\mathrm{kIQR}$ & $\%$ tile & $\mathrm{kIQR}$ & $\%$ tile & kIQR & \\
\hline AKT1 & 2 & 2 & -1.37 & 0 & -1.70 & 0 & -1.49 & -1.01 \\
\hline$A P 4 B 1$ & 1 & 25 & -0.32 & 50 & -0.16 & 4 & -0.46 & +1.04 \\
\hline$A R$ & 3 & 75 & +0.89 & 54 & +0.10 & 79 & +1.24 & -2.7 \\
\hline ATM & 2 & 48 & -0.03 & 43 & -0.13 & 58 & +0.17 & -1.58 \\
\hline ATRX & 3 & 72 & +0.47 & 60 & +0.17 & 84 & +0.89 & -1.64 \\
\hline BRCA1 & 2 & 35 & -0.25 & 77 & +0.71 & 23 & -0.44 & -1.21 \\
\hline CD27 & 2 & 46 & -0.06 & 52 & 0.04 & 62 & 0.24 & -1.36 \\
\hline CD274 & 2 & 64 & +1.03 & 64 & +3.04 & 76 & +0.29 & +0.28 \\
\hline CHEK2 & 2 & 10 & -0.58 & 29 & -0.35 & 1 & -0.9 & -1 \\
\hline ESR1 & 2 & 88 & +7.84 & 85 & +3.51 & 92 & +11.86 & +6.11 \\
\hline ERBB2 & 2 & 12 & -0.58 & 4 & -0.91 & 20 & -0.62 & +1.13 \\
\hline $\mathrm{FH}$ & 1 & 2 & -0.9 & 0 & -0.58 & 7 & -0.71 & -2.47 \\
\hline HMGA2 & 2 & 95 & +7.71 & 100 & +213.35 & 93 & +6.79 & +7.47 \\
\hline IGF2 & 2 & 90 & +6.63 & 89 & +2.91 & 69 & +0.32 & +2.49 \\
\hline IGF2BP2 & 2 & 62 & +0.31 & 94 & +2.21 & 72 & +0.74 & +2.38 \\
\hline JAK1 & 2 & 98 & +3.11 & 99 & +2.42 & 100 & +3.85 & -1.12 \\
\hline KIT & 2 & 79 & +1.07 & 37 & -0.29 & 81 & +1.30 & -1.37 \\
\hline MED12 & 3 & 80 & +0.73 & 92 & +1.19 & 92 & 1.21 & +1.67 \\
\hline MDM2 & 2 & 71 & +0.45 & 90 & +1.19 & 74 & +0.64 & +1.15 \\
\hline MDM4 & 3 & 85 & +1.08 & 97 & +1.89 & 96 & +1.89 & -1.47 \\
\hline NFE2L2 & 2 & 70 & +0.42 & 40 & -0.17 & 81 & +0.73 & +1.12 \\
\hline PLAG1 & 2 & 94 & +2.93 & 100 & +5.6 & 94 & +7.94 & +2.48 \\
\hline$P G R$ & 2 & 93 & +10.18 & 84 & +2.28 & 95 & +3.93 & +1.85 \\
\hline RAD51B & 0 & 15 & -0.59 & 15 & -0.61 & 14 & -0.58 & -1.13 \\
\hline TP53 & 2 & 31 & -0.29 & 60 & +0.14 & 55 & +0.09 & +1.06 \\
\hline
\end{tabular}

All TCGA, All TCGA (The Cancer Genome Atlas) cancers data set; SARC, sarcoma data set from TCGA; Body Map 2.0, Illumina Human Body Map 2.0; \%ile, percentile; KIQR, number of interquartile range intervals away from the median; FC, fold change in gene expression.

(q14.3;q24.1)(g.65999147;g.68758275) event disrupting RAD51B in intron 7 (NM_133510.3, ENST00000471583) on Chromosome 14 (Fig. 3C,D). Neither structural variant appears subclonal given the amount of supporting reads observed (Fig. 3; Supplemental Table S1). We can deduce that these structural variants occur on both alleles because they overlap. Therefore, given that the region containing RAD51B falls in is copy neutral (two copies), all copies of RAD51B have been disrupted and a functional protein product is unlikely (Fig. 3). Expression of RAD51B was low by percentile (Table 1), but not an extreme outlier according to the TCGA tumor distribution and normal tissue comparison (Table 1). Given the estimated tumor content (42\%), these expression values are probably artificially inflated by contribution of the normal tissue. The translocation breakpoint on Chromosome 12 lands 
A

B
Deletion

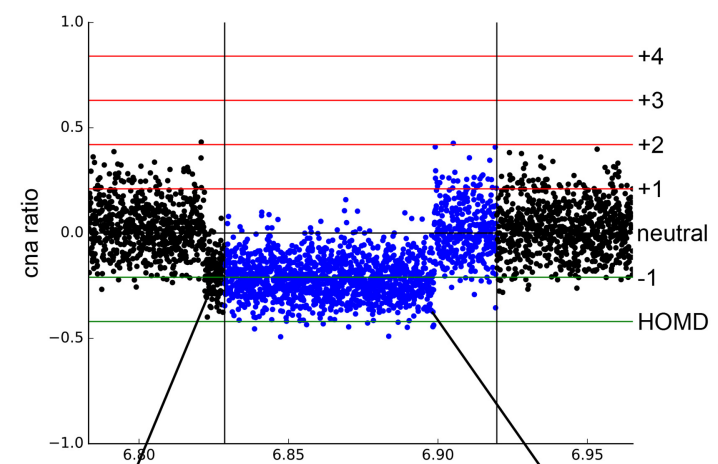

C

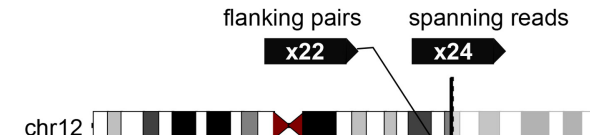

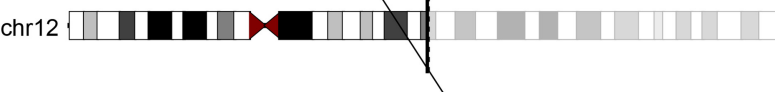

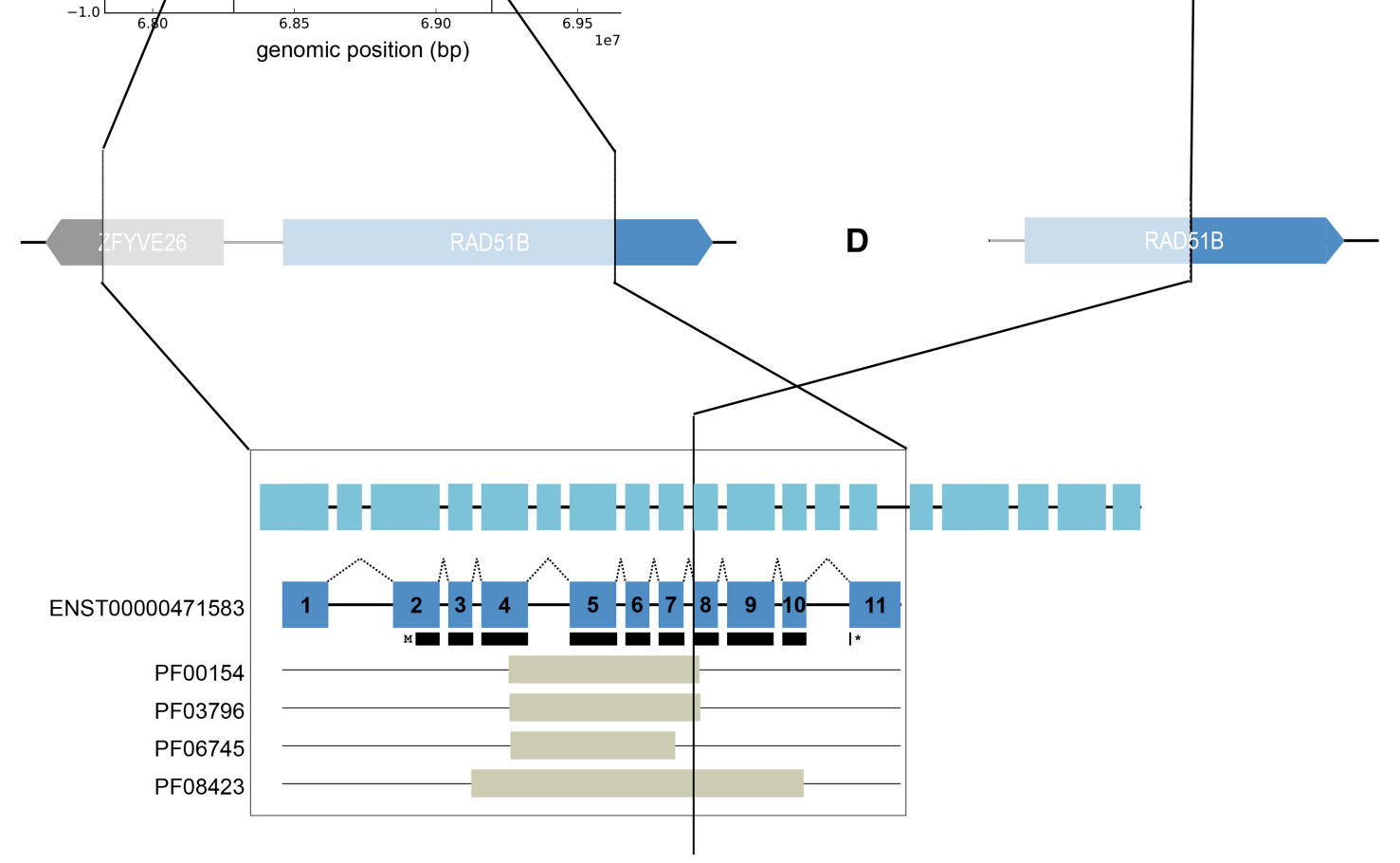

Figure 3. Structural variants disrupting both alleles of the RAD51B gene. (A) Copy-number data supporting the Chr14:g.68219215_68986637del (767,422-bp) deletion. The event was called using DELLY, demonstrating the presence of flanking reads pairs (12 paired-end reads) and spanning reads (14 split reads) supporting the deletion breakpoints. Copy number alteration (cna) ratio is plotted on the $y$-axis and genomic position is plotted on the $x$-axis. The horizontal lines represent the expected value for different copy states using the $42 \%$ diploid model ( 0 is copy neutral, -1 represents a heterozygous copy loss, and HOMD refers to homozygous deletion copy loss). Data points are colored blue if they fall within RAD51B. (B) A gene-level overview of the same deletion. (C) A chromosome-level overview of the translocation $t(12 ; 14)$ (g.65999147; 9.68758275$)$. The event was called using Trans-ABySS, demonstrating the presence of flanking read pairs (22 paired-end reads) and spanning reads (24 split reads) supporting the translocation breakpoint. (D) A gene-level overview of the same translocation breakpoint within RAD51B. (E) A transcript-level overview of all RAD51B breakpoints from both the translocation and deletion events. The top track shows the collapsed exons from all transcripts (ENSG00000182185). Translation start and end are displayed by the black discontinuous rectangle directly below the exons of the selected transcript (ENST00000471583). Domains are given by the bottom tracks and are labeled by Pfam identifiers: RecA (PF00154); DnaB-like helicase carboxy-terminal domain (PF03796); KaiC (PF06745); Rad51 (PF08423). 
COLD SPRING HARBOR Molecular Case Studies
Leiomyosarcoma genomic profiling and CHEK2 variant

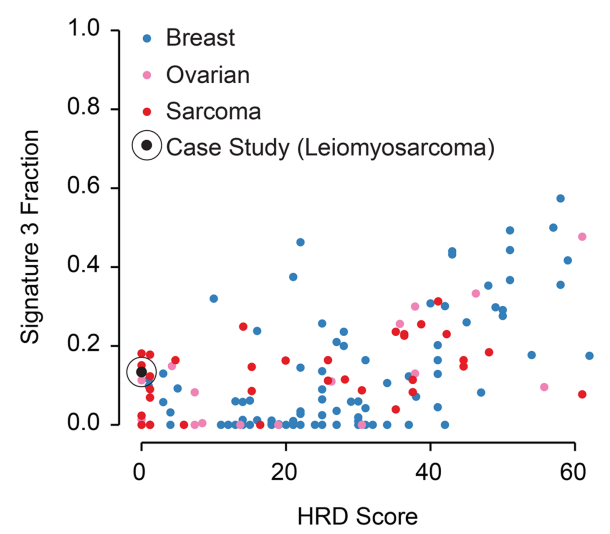

Figure 4. Homologous recombination deficiency (HRD) score and signature 3 exposure levels (indicators of HRD status). The HRD score of the case study was 0 (circled black point), indicating that there was no large loss of heterozygosity, telomeric allelic imbalance, or large-scale transition events. Signature 3, associated with HRD, accounted for $13.4 \%$ of total somatic single-nucleotide variant burden. Shown here is a comparison of these two indicators of HRD status against 139 other cancers (36 sarcomas, 87 breast cancers, and 16 ovarian cancers).

$219 \mathrm{~kb}$ upstream of HMGA2 (12q14.3), and HMGA2 expression was very elevated when compared with all TCGA cancers and TCGA sarcomas (Table 1).

Increased expression of PLAG1 and IGF2 was also noted (Table 1). There was a copy loss of $F H$, and $F H$ appeared down-regulated in our patient's LMS (second percentile for all TCGA cancers). Interestingly enough, FH mutations lead to a hereditary cancer predisposition: hereditary leiomyomatosis renal cell carcinoma syndrome. Therefore, we pursued further investigation regarding $\mathrm{FH}$ status given the potential clinical implications and possibility to tailor oncological management. However, FH immunochemistry revealed positive staining in keeping with the protein being present (data not shown).

A duplication involving the majority of the Chromosome $X$ long arm leads to ATRX (Xq21.1) and MED12 (Xq13.1) copy gains. ATRX and MED12 loss-of-function variants have been implicated in leiomyoma and LMS pathophysiology (Discussion and Supplemental Discussion), but no such variants were seen in our sample.

\section{Mutational Signature Analysis}

Cancers have specific recurrent mutational signatures (patterns) associated with their underlying somatic molecular pathogenesis and these signatures guide the interpretation of genomic events leading to tumorigenesis (Alexandrov et al. 2013). The mutational signature correlating with BRCA1/BRCA2 loss (signature 3) (http://cancer.sanger.ac.uk/ cosmic/signatures) accounted for $<20 \%(13.4 \%, 95 \% \mathrm{Cl} 6.2 \%-19.1 \%)$ of mutational burden (Supplemental Table S2; Supplemental Figs. S3, S4). This fell in the 50th percentile among 36 sarcomas, 69th percentile among 16 ovarian cancers, and 69th percentile among 87 breast cancers (Fig. 4). However, the calculated homologous recombination deficiency score was 0 , because of a lack of large-scale LOH, TAI (telomeric allelic imbalance), and LST (large-scale state transition) events. Please refer to Methods and Supplemental Methods for more details.

\section{DISCUSSION}

In summary, we describe the findings of genomic profiling in an LMS patient with a germline CHEK2:c.1100delC variant and concomitant diagnosis of metastatic invasive ductal breast 
COLD SPRING HARBOR Molecular Case Studies
Leiomyosarcoma genomic profiling and CHEK2 variant carcinoma. Of the known recurrent mutations and large rearrangements observed in leiomyomas and LMSs (Supplemental Discussion), the analysis of our patient's tumor revealed a translocation $\mathrm{t}(12 ; 14)$ with one breakpoint disrupting RAD51B and the other breakpoint upstream of HMGA2 (Mehine et al. 2013; Bertsch et al. 2014), a large 16q deletion encompassing FANCA (reported in two cases) (Beck et al. 2009) and nonspecific copy gains and losses on Chromosome 1 (e.g., telomeric loss of 1p) (Packenham et al. 1997; Ul-Hassan et al. 2009).

The molecular mechanisms underlying the impact of germline CHEK2 pathogenic variants in relation to tumorigenesis have not been elucidated. Somatic CHEK2 point mutations have been identified in osteosarcomas and also rarely in lung and ovarian tumors, but not in other types of sarcomas tested in one study (Miller et al. 2002). Despite the involvement of CHEK2 protein in double-strand DNA break (DSB) repair, unlike BRCA1/BRCA2-driven tumors, somatic inactivation of the wild-type allele is uncommon in breast tumors of CHEK2 mutation carriers (Suspitsin et al. 2014). CHEK2 is part of a complex network of activating cascades, which is mainly regulated through phosphorylation events. The initial cellular event triggering CHEK2 activation is its phosphorylation by ataxia telangiectasia mutated (ATM) in the context of DNA damage (Zannini et al. 2014). Activated CHEK2 then phosphorylates BRCA1 and BRCA2, which in return promotes homology-directed repair, a more accurate DSB repair mechanism when compared with nonhomologous end joining (Zannini et al. 2014). In vivo studies have showed that CHEK2:c.1100delC impairs CHEK2 kinase activity and affects its oligomerization and autophosphorylation ( $X u$ et al. 2002). Therefore, CHEK2-mediated BRCA1/BRCA2 (Zannini et al. 2014) and TP53 (Hirao et al. 2000) phosphorylation may be impaired. Also, this mutation may affect CHEK2 phosphorylation by ATM, a gene frequently deleted in LMS tumors (Ul-Hassan et al. 2009). In our patient's tumor, the homologous recombination deficiency score was 0 and BRCA1/BRCA2 mutational signature (signature 3) was modest compared with tumors with a characterized loss of BRCA1/BRCA2 functionality (Alexandrov et al. 2013). Overall, there was no evidence of double-strand DNA repair deficiency in the LMS.

\section{Genomic Analysis-Discussion}

RAD51 recombinase has a central role in homologous recombination and double-strand DNA (dsDNA) break repair (Suwaki et al. 2011). RAD51B protein is part of the BCDX2 complex (RAD51B, RAD51D, XRCC2), which recruits RAD51 protein to damage foci (Suwaki et al. 2011). Mild RAD51B haploinsufficiency was shown to cause a minor increase of sensitivity to DNA-damaging agents in human colon cancer cell lines, whereas more severe RAD51B impairment has been associated with centrosome fragmentation and aneuploidy (Date et al. 2006). Recurrent translocations involving both RAD51B (14q24.1) and HMGA2 (12q14.3) loci play an important role in the pathogenesis of uterine leiomyoma via impaired cell-cycle checkpoint control and deficient dsDNA repair mechanisms (Mehine et al. 2013). Chromosome 12 breakpoints in such rearrangements have been shown as intragenic or extragenic, the latter being observed in the current case with a breakpoint $219 \mathrm{~kb}$ upstream of HMGA2 (Quade et al. 2003; Medeiros et al. 2010). HMGA2 rearrangements make up a unique subtype of leiomyomas, which also feature overexpression of specific genes: IGF2BP2, PLAG1, IGF2, HMGA2 (Mehine et al. 2016). This is consistent with the pattern of expression observed in our LMS, with the exception of IGF2BP2, which displayed average expression (Table 1).

Uterine leiomyomas are benign smooth muscle tumors and their potential to progress to LMS is still an area of debate in the literature (Di Luigi et al. 2015). The acquisition of a TP53 mutation appears to be a frequent and critical difference between leiomyoma and LMS (de Vos et al. 1994). Somatic MED12 alterations are involved in the pathophysiology of both leiomyomas (66\%) and LMSs (20\%) (Pérot et al. 2012). Somatic ATRX mutations are 
thought to participate in LMS oncogenesis via impaired telomere-maintenance mechanisms (Yang et al. 2015). Mäkinen et al. (2016) performed whole-exome sequencing in 19 LMSs and found several somatic TP53, MED12, and ATRX mutations, therefore reiterating the central role of these genes in a subset of LMSs (Mäkinen et al. 2016). These genes did not harbor somatic loss-of-function mutations nor aberrant expression in our patient's LMS (Table 1).

Even though $\mathrm{FH}$ expression appeared low in conjunction with a somatic $\mathrm{FH}$ copy loss, $\mathrm{FH}$ immunochemistry revealed positive staining in keeping with the presence of the protein. This result does not support a central role for FH deficiency in the genesis of our patient's LMS, which is essentially in keeping with the leiomyoma subtype with HMGA2 aberrations (Mehine et al. 2016). Somatic FH deletion is not an uncommon event in leiomyomas and LMSs, but the exact role of this genomic alteration in smooth muscle tumor malignant progression requires further study (Liegl-Atzwanger et al. 2016).

\section{Therapeutic Implications-CHEK2}

BRCA1/BRCA2-driven breast cancers show enhanced response to DNA-damaging therapies such as platinum agents and poly ADP ribose polymerase (PARP) inhibitors, but reduced response to anthracycline-taxane chemotherapy regimens (Smith and Isaacs 2011). Even though CHEK2 mutation carriers display a particularly poor response to neoadjuvant therapy with anthracycline alone (without taxane) (Pfeifer et al. 2014), there has been no study focusing on platinum or PARP inhibitor response within the CHEK2 mutation carrier breast cancer group.

Functional studies of high-grade serous ovarian cancer show that low CHEK2 expression surprisingly correlates with poorer platinum response and CHEK2 depletion leads to platinum resistance by suppressing cisplatin-induced S-phase cell cycle arrest (Alkema et al. 2014). This would suggest that CHEK2 haploinsufficiency could be a contraindication to platinum-based therapy in these patients.

Unlike BRCA1/BRCA2-driven breast cancer cases, CHEK2 mutation carriers more frequently present with HR-positive tumors (Atchley et al. 2008; Weischer et al. 2012). However, how to incorporate this clinicopathological tumor feature into the context of CHEK2 mutation carrier hormonal therapy still remains uncertain. To date, small casecontrol studies report no significant difference between the response of CHEK2 mutation carriers to hormonal therapy when compared with noncarriers (Kriege et al. 2014, 2015).

Churpek et al. (2016) found a significantly higher incidence of inherited cancer gene mutations in their breast cancer cohort developing treatment-related leukemia, and even though only one case of CHEK2 mutation was identified, this new association carries therapeutic implications for both cancer treatment and monitoring of secondary complications (Churpek et al. 2016). Posttreatment, our patient did not present hematopoietic abnormalities suggestive of hematological malignancy.

\section{Therapeutic Implications_-Germline and Somatic Genomic Profiling}

Theoretically, some aspects of the genomic profiling results in this patient could suggest a platinum agent response and PARP inhibitor response: RAD51B alterations, very high HMGA2 expression, and germline CHEK2 pathogenic mutation. A recent review of literature by Ward et al. (2015) on preclinical RAD51 targeting agents supports the therapeutic role of RAD51 inhibitors (likely combined with PARP inhibitors) to impair homologous recombination DNA repair and therefore induce cancer radiation and chemotherapy sensitization (Ward et al. 2015). We would therefore hypothesize that biallelic RAD51B disruption, such as present in our patient, may be a good prognostic factor. However, we did not identify a strongly damaging mutation or expression outlier in the DSB repair pathway. Moreover, despite the presence of several mutations in the DSB repair pathway genes and previous 
COLD SPRING HARBOR Molecular Case Studies
Leiomyosarcoma genomic profiling and CHEK2 variant exposure to radiation therapy, a well-known dsDNA break inducer (Thompson 2012), the mutational burden was unremarkable and mutational signature 3, associated with HRD and BRCA1/BRCA2 loss, accounted for <20\% (13.4\%, 95\% Cl 6.2\%-19.1\%) of total somatic single-nucleotide variant burden. Consequently, although some aspects of our data suggest that multiple hits may potentiate DSB repair deficiency in the presence of DNA-damaging therapies and favor synthetic lethality, the current literature does not support this approach because of the lack of evidence-based studies on small effect hits in the DSB repair pathway and their clinical implications. Given the current conflicting literature on this topic, paucity of published therapeutic trials, and possible risk of harm, DNA-damaging therapy can only be considered in the setting of a clinical trial.

The hormonal status of uterine LMSs varies widely among different literature sources: ER immunohistochemistry (IHC)-positive in 18\%-87\% of cases (Amant et al. 2009) and androgen receptor (AR)-positive in 40\%-100\% of cases (Leitao et al. 2004; Koivisto-Korander et al. 2011). Some data suggest that ER-positive LMS status is associated with a better prognosis (loffe et al. 2009; Koivisto-Korander et al. 2011). Clinical response to aromatase inhibitor therapy in advanced LMS is rare and has been observed solely in ER-positive tumors, with which our patient presents (Hardman et al. 2007; loffe et al. 2009; O'Cearbhaill et al. 2010). Of note, our patient has a diagnosis of PCOS and several studies have reported increased risks of breast, endometrial, and ovarian cancer in PCOS patients (Chittenden et al. 2009; Daniilidis and Dinas 2009). A recent meta-analysis found a significant increased risk only for endometrial cancer (odds ratio [OR] 2.79, Cl 1.31-5.95) overall, but subgroup analysis excluding women over age 54 revealed that risk of both endometrial (OR 4.05, $\mathrm{Cl}$ 2.42-676) and ovarian (OR 2.52, Cl 1.08-5.89) cancer was increased (Barry et al. 2014).

\section{Conclusion}

This is the first report of LMS genomic profiling in a patient with a germline CHEK2: c. 1100delC variant and concomitant diagnosis of metastatic invasive ductal breast carcinoma. Our findings suggest that pathogenic germline CHEK2 mutations may increase the risk of several types of malignancies, but that complete tumor genome and transcriptome analysis does not necessarily demonstrate obvious impairment of DSB repair mechanisms. We report a new possible mechanistic relationship between leiomyoma and LMS based on genomic and transcriptome data. Our results suggest that translocations with one breakpoint disrupting RAD51B and the second breakpoint upstream of HMGA2 represent a genomic subtype of genital type LMS sharing similarities with leiomyoma. Both RAD51B alleles were disrupted, as one is involved in the translocation and the second is almost completely removed through a 767,422-bp deletion. Given the disparity between uterine leiomyoma prevalence and LMS incidence, malignant progression is thought to be rare. Our data support a role for RAD51B structural events associated with HMGA2 overexpression in oncogenesis and potentially promoting the transition from leiomyoma to LMS. Future detailed genomic profiling studies are required to inform tailored treatment of such tumors and the optimal management of cancer in patients with a germline CHEK2 mutation remains to be elucidated. Functional assessment of protein expression, such as detailed pathology review, immunochemistry testing, and proteomics, complement whole-genome and transcriptome profiling and will contribute to enhance our understanding of cancer pathogenesis.

\section{METHODS}

\section{Sample Collection, RNA and DNA Extraction, and Library Preparation}

Following informed consent, the patient underwent imaging-guided biopsies as part of the Personalized OncoGenomics program of British Columbia. An incisional biopsy of a 
15-cm-diameter tumor from the pelvic genital type LMS was obtained. Up to eight core biopsies were collected on two separate occasions using 18-22G biopsy needles. Peripheral venous blood samples were obtained at the time of biopsy and leukocytes isolated for germline DNA analysis. DNA and RNA extraction, library construction, and sequencing were performed according to standard protocols (Jamshidi et al. 2014). Please refer to Supplemental Methods for more details.

\section{Sequencing and Bioinformatics}

Paired-end reads (125 bp for genome sequencing, 75 bp for transcriptome sequencing) were generated on an Illumina HiSeq2500 sequencer (Illumina Inc.; http://www.illumina. com/). Tumor biopsy and peripheral blood samples were sequenced to a depth of $93 \times$ and $46 \times$, respectively, using established polymerase chain reaction (PCR)-free wholegenome sequencing and strand-specific RNA-seq protocols previously described (Jones et al. 2016). More than 267 million passed filter reads were obtained from the RNA-seq experiment. Reads were aligned to the hg19 (GRCh37) reference using BWA-align v0.5.7 (Li and Durbin 2010). For both germline and somatic genomic events, variant calling and filtering was performed with mpileup and varFilter from SAMtools (v0.1.17), respectively (Li et al. 2009; Sheffield et al. 2016). The CHEK2:c.1100delC variant was reviewed and validated by Sanger sequencing in the British Columbia Cancer Agency clinical cytogenetics laboratory.

Tumor content and ploidy model were estimated from sequencing data through analysis of the CNA (copy-number alterations) ratios and allelic frequencies of each chromosome (Jones et al. 2010; Ha et al. 2012). The tumor and matched normal samples were analyzed in conjunction to identify LOH and CNVs with CNAseq (v0.0.6) (Jones et al. 2010) and APOLLOH (v0.1.1) (Ha et al. 2012), respectively. The collection of distinct CNA and loss of $\mathrm{LOH}$ regions was compared with a set of theoretical models for ploidy (ranging from diploid to pentaploid) and tumor content (10\% intervals from the initial estimated tumor content: $12 \%, 22 \%, 32 \%, 42 \%, 52 \%, 62 \%, 72 \%, 82 \%, 92 \%)$. The best fit for the LMS was a diploid model at $42 \%$ tumor content, which is a lower tumor content than estimated via pathology review and assessment (72.5\%).

A combination of tools was used to perform somatic SNV calling: SAMtools (v0.1.17) (Li et al. 2009), MutationSeq (v1.0.2) (Ding et al. 2012), and Strelka (v1.0.6) (Saunders et al. 2012); small insertions and deletions were identified using Strelka (v1.0.6) (Saunders et al. 2012) and Trans-ABySS (v1.4.10) (Birol et al. 2009; Simpson et al. 2009). Small-mutation (SNV, indel) calling takes into consideration the tumor content (42\%) and ploidy model (diploid), which corrects for copy-number changes and their effect on variant calling. De novo assembly and annotation of genomic and transcriptomic data using ABySS (v1.3.4) (Robertson et al. 2010), Trans-ABySS (v1.4.10) (Birol et al. 2009; Simpson et al. 2009), and DELLY v0.6.1 (Rausch et al. 2012) was utilized to identify structural variants and fusion genes.

The somatic variants were then used to examine mutational signatures/patterns (Alexandrov et al. 2013).

RNA expression was analyzed with respect to publically available RNA-seq tumor data sets: The Cancer Genome Atlas (TCGA) Research Network (http://cancergenome.nih.gov/) and Illumina Human Body Map 2.0 RNAseq (16 different human tissues). The Illumina Human Body Map 2.0 data can be accessed from ArrayExpress (query \#E-MTAB-513). Gene-based RPKM (transcript-normalized) values were calculated using Ensembl (v69) gene models. To compare to TCGA data sets, a mapping from a given Ensembl gene to the appropriate TGCA gene was determined based on position overlap using the TCGA Generic Annotation File (GAF) files. Several metrics were calculated: percentile, fold change 
COLD SPRING HARBOR Molecular Case Studies
Leiomyosarcoma genomic profiling and $\mathrm{CHEK} 2$ variant (over the mean), and kIQR. The kIQR is defined as the number of interquartile range intervals away from the median. Given the diagnosis, the TCGA SARC data set (tumor and matched normal) was chosen as the primary comparators and the compendium average of the tissues in the Illumina Human Body Map RNA-seq data set was used as an additional normal tissue comparator. Results were also compared to the full compendium of TCGA cancers data set. For additional details on the sequencing data analytical pipeline used, please refer to Supplemental Methods.

\section{Calculation of Mutation Signature Exposures}

Somatic SNVs derived from whole-genome sequencing were classified into 96 mutation types representing the base substitution and trinucleotide $5 / 13^{\prime}$ context using SomaticSignatures (Gehring et al. 2015). The mutation signature model, as previously described (Alexandrov et al. 2013), takes the form

$$
\mathrm{m}=\mathrm{Pe},
$$

where $\mathbf{m}$ is a 96-element vector enumerating the mutations per class, $\mathbf{P}$ is a $96-$-by-30 matrix denoting the relative mutation class frequencies in 30 previously described signatures (available at http://cancer.sanger.ac.uk/cosmic/signatures), and e is the 30-element exposure that represents the number of mutations contributed by each of the 30 associated mutational processes. As per Alexandrov et al. (2015), e was computed using nonnegative least squares (Alexandrov et al. 2015). Monte Carlo resampling was performed to determine the $95 \%$ confidence interval of each signature's contribution to the overall mutational burden (Supplemental Methods; Supplemental Table S2; Supplemental Figures S3, S4).

However, because of the lack of previous study of mutation signatures in LMS, we did not limit the signature space to a subset of possible solutions. The exposure of signature 3 was taken to be the homologous recombination deficiency signature.

\section{Determination of Homologous Recombination Deficiency Score}

The HRD score was determined based on guidelines in previous studies (Timms et al. 2014; Telli et al. 2015), adapted to whole-genome sequencing data. The HRD score was calculated as an arithmetic sum of HRD-LOH, HRD-TAI, and HRD-LST scores. HRD-LOH was defined as the number of regions of $\mathrm{LOH}>15 \mathrm{Mb}$ in length, but smaller than the whole chromosome. HRD-TAl was defined as the number of regions of allelic imbalance extending to one subtelomere but not across the centromere. Here, a subtelomere was defined as the region between the telomeric boundary and the closest gene, minus a 10-kb flanking region. HRD-LST was defined as the number of breakpoints between regions of $>10 \mathrm{Mb}$ after filtering out regions of $<3 \mathrm{Mb}$.

\section{Comparison of HRD Scores and Signatures}

The HRD score and HRD mutation signature were compared against those calculated for 87 breast cancers, 16 ovarian cancers, and 36 sarcomas, sequenced at our Genome Sciences Centre.

\section{ADDITIONAL INFORMATION}

\section{Data Deposition and Access}

Whole-genome sequencing and RNA-seq data (.bam files) have been submitted to the European Genome-Phenome Archive (EGA) (www.ebi.ac.uk/ega/home) under data 
C OLD SPR ING HARBOR Molecular Case Studies
Leiomyosarcoma genomic profiling and CHEK2 variant accession number EGAD00001002891. The CHEK2:c.1100delC variant has been submitted to ClinVar (https://www.ncbi.nlm.nih.gov/clinvar/) under accession number SCV000574565.

\section{Ethics Statement}

The patient specimens were collected as part of a research project approved by the University of British Columbia Cancer Agency (BCCA) Research Ethics Board (REB) (protocol $\mathrm{H14-00681)}$ and are in accordance with the Declaration of Helsinki. Informed written consent was obtained from the patient for tumor profiling using RNA-seq (tumor) as well as wholegenome sequencing (tumor and blood). The use of data sets is allowed for research reports and scientific publications. The protocol allows for data to be not only used for research reports and scientific publication but also to be made available to named investigators of institutions who agree by a data transfer agreement stating they will honor the same ethical and privacy principles required by the BCCA REB.

\section{Acknowledgments}

We gratefully acknowledge the participation of the patient. The authors are grateful for the funding support provided by the British Columbia Cancer Agency Foundation. We thank Payal Sipahimalani, Jessica Nelson, Alexandra Fok, and Robyn Roscoe for project management. We thank Payal Sipahimalani and Patrick Plettner for genomic data deposition. We thank Erin Pleasance for her useful comments and suggestions. We thank Morgan Bye and Nina Thiessen for providing further information on the bioinformatic pipeline. We thank Martin Jones for facilitating manuscript submission.

\section{Author Contributions}

S.J.M.J., K.A.S., M.A.M., and J.L. contributed to conception and design of this study. L.A.M. and R.T. provided patient clinical data and oncological management information for this study. Clinical data were reviewed by L.A.M., K.A.S., S.S., K.G., H.J.L., and D.J.R. Pathological study was contributed by M.H., S.Y., T.N., and Y.A. Data production, analysis, and interpretation was contributed by Y.Z., A.J.M., K.L.M, R.A.M., A.K., C.R., C.C., and M.L.T. Detailed Methods description was contributed by A.J.M. and Y.P.M. Germline assessment was contributed by S.S.Y. Homologous recombination deficiency analysis and Monte Carlo resampling were contributed by E.Z. Manuscript writing and critical editing was contributed by M.L.T., C.R., E.Z., K.A.S., and S.J.M.J. All authors approved the final manuscript.

\section{Funding}

This research was generously funded through unrestricted philanthropic donations received through the BC Cancer Foundation (NRF10229). This work was also supported by the Terry Fox Foundation Program Project Grant funding held by A.K. (TFF-122869), the Michael Smith Foundation for Health Research held by K.A.S., and the Canadian Institutes of Health Research held by K.A.S. M.A.M. acknowledges infrastructure investments from the Canada Foundation for Innovation and the support of the Canada Research Chairs and ClHR Foundation (FDN-143288) programs.

\section{Clinical Trial}

This research was conducted as part of the Personalized OncoGenomics (POG) clinical trial in British Columbia, Canada (NCT02155621). 


\section{REFERENCES}

Alexandrov LB, Nik-Zainal S, Wedge DC, Aparicio SAJR, Behjati S, Biankin AV, Bignell GR, Bolli N, Borg A, Børresen-Dale A-L, et al. 2013. Signatures of mutational processes in human cancer. Nature 500: 415-421.

Alexandrov LB, Jones PH, Wedge DC, Sale JE, Campbell PJ, Nik-Zainal S, Stratton MR. 2015. Clock-like mutational processes in human somatic cells. Nat Genet 47: 1402-1407.

Alkema NG, Tomar T, van der Zee AGJ, Everts M, Meersma GJ, Hollema H, de Jong S, van Vugt MATM, Wisman GBA. 2014. Checkpoint kinase 2 (Chk2) supports sensitivity to platinum-based treatment in high grade serous ovarian cancer. Gynecol Oncol 133: 591-598.

Amant F, Coosemans A, Debiec-Rychter M, Timmerman D, Vergote I. 2009. Clinical management of uterine sarcomas. Lancet Oncol 10: 1188-1198.

Atchley DP, Albarracin CT, Lopez A, Valero V, Amos Cl, Gonzalez-Angulo AM, Hortobagyi GN, Arun BK. 2008. Clinical and pathologic characteristics of patients with BRCA-positive and BRCA-negative breast cancer. $J$ Clin Oncol 26: 4282-4288.

Barry JA, Azizia MM, Hardiman PJ. 2014. Risk of endometrial, ovarian and breast cancer in women with polycystic ovary syndrome: a systematic review and meta-analysis. Hum Reprod Update 20: 748-758.

Beck AH, Lee CH, Witten DM, Gleason BC, Edris B, Espinosa I, Zhu S, Li R, Montgomery KD, Marinelli RJ, et al. 2009. Discovery of molecular subtypes in leiomyosarcoma through integrative molecular profiling. Oncogene 29: 845-854.

Bell DW, Varley JM, Szydlo TE, Kang DH, Wahrer DC, Shannon KE, Lubratovich M, Verselis SJ, Isselbacher KJ, Fraumeni JF, et al. 1999. Heterozygous germ line hCHK2 mutations in Li-Fraumeni syndrome. Science 286: $2528-2531$

Bertsch E, Qiang W, Zhang Q, Espona-Fiedler M, Druschitz S, Liu Y, Mittal K, Kong B, Kurita T, Wei J-J. 2014. MED12 and HMGA2 mutations: two independent genetic events in uterine leiomyoma and leiomyosarcoma. Mod Pathol 27: 1144-1153.

Birol I, Jackman SD, Nielsen CB, Qian JQ, Varhol R, Stazyk G, Morin RD, Zhao Y, Hirst M, Schein JE, et al. 2009. De novo transcriptome assembly with ABySS. Bioinformatics 25: 2872-2877.

Chittenden BG, Fullerton G, Maheshwari A, Bhattacharya S. 2009. Polycystic ovary syndrome and the risk of gynaecological cancer: a systematic review. Reprod BioMed Online 19: 398-405.

Churpek JE, Marquez R, Neistadt B, Claussen K, Lee MK, Churpek MM, Huo D, Weiner H, Bannerjee M, Godley LA, et al. 2016. Inherited mutations in cancer susceptibility genes are common among survivors of breast cancer who develop therapy-related leukemia. Cancer 122: 304-311.

Daniilidis A, Dinas K. 2009. Long term health consequences of polycystic ovarian syndrome: a review analysis. Hippokratia 13: 90-92.

Date O, Katsura M, Ishida M, Yoshihara T, Kinomura A, Sueda T, Miyagawa K. 2006. Haploinsufficiency of RAD51B causes centrosome fragmentation and aneuploidy in human cells. Cancer Res 66: 6018-6024.

Desrichard A, Bidet Y, Uhrhammer N, Bignon Y-J. 2011. CHEK2 contribution to hereditary breast cancer in non-BRCA families. Breast Cancer Res 13: R119.

de Vos S, Wilczynski SP, Fleischhacker M, Koeffler P. 1994. p53 alterations in uterine leiomyosarcomas versus leiomyomas. Gynecol Oncol 54: 205-208.

Di Luigi G, D'Alfonso A, Patacchiola F, Di Stefano L, Palermo P, Carta G. 2015. Leiomyosarcoma: a rare malignant transformation of a uterine leiomyoma. Eur J Gynaecol Oncol 36: 84-87.

Ding J, Bashashati A, Roth A, Oloumi A, Tse K, Zeng T, Haffari G, Hirst M, Marra MA, Condon A, et al. 2012. Feature-based classifiers for somatic mutation detection in tumour-normal paired sequencing data. Bioinformatics 28: 167-175.

Gehring JS, Fischer B, Lawrence M, Huber W. 2015. SomaticSignatures: inferring mutational signatures from single-nucleotide variants. Bioinformatics 31: 3673-3675.

Ha G, Roth A, Lai D, Bashashati A, Ding J, Goya R, Giuliany R, Rosner J, Oloumi A, Shumansky K, et al. 2012. Integrative analysis of genome-wide loss of heterozygosity and monoallelic expression at nucleotide resolution reveals disrupted pathways in triple-negative breast cancer. Genome Research 22: 1995-2007.

Hardman MP, Roman JJ, Burnett AF, Santin AD. 2007. Metastatic uterine leiomyosarcoma regression using an aromatase inhibitor. Obstet Gynecol 110: 518-520.

Hirao A, Kong Y-Y, Matsuoka S, Wakeham A, Ruland J, Yoshida H, Liu D, Elledge SJ, Mak TW. 2000. DNA damage-induced activation of p53 by the checkpoint kinase Chk2. Science 287: 1824-1827.

loffe YJ, Li AJ, Walsh CS, Karlan BY, Leuchter R, Forscher C, Cass I. 2009. Hormone receptor expression in uterine sarcomas: prognostic and therapeutic roles. Gynecol Oncol 115: 466-471.

Jamshidi F, Pleasance E, Li Y, Shen Y, Kasaian K, Corbett R, Eirew P, Lum A, Pandoh P, Zhao Y, et al. 2014. Diagnostic value of next-generation sequencing in an unusual sphenoid tumor. Oncologist 19: 623-630.

Jones SJ, Laskin J, Li YY, Griffith OL, An J, Bilenky M, Butterfield YS, Cezard T, Chuah E, Corbett R, et al. 2010. Evolution of an adenocarcinoma in response to selection by targeted kinase inhibitors. Genome Biol 11: R82. 
Jones MR, Schrader KA, Shen Y, Pleasance E, Ch'ng C, Dar N, Yip S, Renouf DJ, Schein JE, Mungall AJ, et al. 2016. Response to angiotensin blockade with irbesartan in a patient with metastatic colorectal cancer. Ann Oncol 27: 801-806.

Koivisto-Korander R, Butzow R, Koivisto A-M, Leminen A. 2011. Immunohistochemical studies on uterine carcinosarcoma, leiomyosarcoma, and endometrial stromal sarcoma: expression and prognostic importance of ten different markers. Tumor Biol 32: 451-459.

Koivisto-Korander R, Martinsen JI, Weiderpass E, Leminen A, Pukkala E. 2012. Incidence of uterine leiomyosarcoma and endometrial stromal sarcoma in Nordic countries: results from NORDCAN and NOCCA databases. Maturitas 72: 56-60.

Kriege M, Hollestelle A, Jager A, Huijts PEA, Berns EM, Sieuwerts AM, Meijer-van Gelder ME, Collée JM, Devilee P, Hooning MJ, et al. 2014. Survival and contralateral breast cancer in CHEK2 1100delC breast cancer patients: impact of adjuvant chemotherapy. Br J Cancer 111: 1004-1013.

Kriege M, Jager A, Hollestelle A, Berns EMJJ, Blom J, Meijer-van Gelder ME, Sieuwerts AM, van den Ouweland A, Collée JM, Kroep JR, et al. 2015. Sensitivity to systemic therapy for metastatic breast cancer in CHEK2 1100delC mutation carriers. J Cancer Res Clin Oncol 141: 1879-1887.

Laskin J, Jones S, Aparicio S, Chia S, Ch'ng C, Deyell R, Eirew P, Fok A, Gelmon K, Ho C, et al. 2015. Lessons learned from the application of whole-genome analysis to the treatment of patients with advanced cancers. Cold Spring Harb Mol Case Stud 1: a000570.

Leedom TP, LaDuca H, McFarland R, Li S, Dolinsky JS, Chao EC. 2016. Breast cancer risk is similar for CHEK2 founder and non-founder mutation carriers. Cancer Genet 209: 403-407.

Leitao MM, Soslow RA, Nonaka D, Olshen AB, Aghajanian C, Sabbatini P, Dupont J, Hensley M, Sonoda Y, Barakat RR, et al. 2004. Tissue microarray immunohistochemical expression of estrogen, progesterone, and androgen receptors in uterine leiomyomata and leiomyosarcoma. Cancer 101: 1455-1462.

Lek M, Karczewski KJ, Minikel EV, Samocha KE, Banks E, Fennell T, O’Donnell-Luria AH, Ware JS, Hill AJ, Cummings BB, etal. 2016. Analysis of protein-coding genetic variation in 60,706 humans. Nature 536:285-291.

Li H, Durbin R. 2010. Fast and accurate long-read alignment with Burrows-Wheeler transform. Bioinformatics 26: 589-595.

Li H, Handsaker B, Wysoker A, Fennell T, Ruan J, Homer N, Marth G, Abecasis G, Durbin R; 1000 Genome Project Data Processing Subgroup. 2009. The Sequence Alignment/Map format and SAMtools. Bioinformatics 25: 2078-2079.

Liegl-Atzwanger B, Heitzer E, Flicker K, Iler SMU, Ulz P, Saglam O, Tavassoli F, Devouassoux-Shisheboran M, Geigl J, Moinfar F. 2016. Exploring chromosomal abnormalities and genetic changes in uterine smooth muscle tumors. Mod Pathol 29: 1262-1277.

Mäkinen N, Aavikko M, Heikkinen T, Taipale M, Taipale J, Koivisto-Korander R, Butzow R, Vahteristo P. 2016. Exome sequencing of uterine leiomyosarcomas identifies frequent mutations in TP53, ATRX, and MED12. PLoS Genet 12: e1005850.

Medeiros F, Araujo AR, Erickson Johnson MR, Kashyap PC, Dal Cin P, Nucci M, Wang X, Bell DA, Oliveira AM. 2010. HMGA1 and HMGA2 rearrangements in mass-forming endometriosis. Genes Chromosomes Cancer 49: 630-634.

Mehine M, Kaasinen E, Mäkinen N, Katainen R, Kämpjärvi K, Pitkänen E, Heinonen H-R, Butzow R, Kilpivaara O, Kuosmanen A, et al. 2013. Characterization of uterine leiomyomas by whole-genome sequencing. N Engl J Med 369: 43-53.

Mehine M, Kaasinen E, Heinonen H-R, Mäkinen N, Kämpjärvi K, Sarvilinna N, Aavikko M, Vähärautio A, Pasanen A, Bützow R, et al. 2016. Integrated data analysis reveals uterine leiomyoma subtypes with distinct driver pathways and biomarkers. Proc Natl Acad Sci 113: 1315-1320.

Meijers-Heijboer H, van den Ouweland A, Klijn J, Wasielewski M, de Snoo A, Oldenburg R, Hollestelle A, Houben M, Crepin E, van Veghel-Plandsoen M, et al. 2002. Low-penetrance susceptibility to breast cancer due to $C H E K 2^{*} 1100$ delC in noncarriers of BRCA1 or BRCA2 mutations. Nat Genet 31: 55-59.

Miller CW, Ikezoe T, Krug U, Hofmann W-K, Tavor S, Vegesna V, Tsukasaki K, Takeuchi S, Koeffler HP. 2002. Mutations of the CHK2 gene are found in some osteosarcomas, but are rare in breast, lung, and ovarian tumors. Genes Chromosomes Cancer 33: 17-21.

Nagel JHA, Peeters JK, Smid M, Sieuwerts AM, Wasielewski M, de Weerd V, Trapman-Jansen AMAC, van den Ouweland A, Brüggenwirth H, van IJcken WFJ, et al. 2012. Gene expression profiling assigns CHEK2 1100 delC breast cancers to the luminal intrinsic subtypes. Breast Cancer Res Treat 132: 439-448.

Näslund-Koch C, Nordestgaard BG, Bojesen SE. 2016. Increased risk for other cancers in addition to breast cancer for CHEK2*1100delC heterozygotes estimated from the Copenhagen general population study. J Clin Oncol 34: 1208-1216.

O'Cearbhaill R, Zhou Q, lasonos A, Soslow RA, Leitao MM, Aghajanian C, Hensley ML. 2010. Treatment of advanced uterine leiomyosarcoma with aromatase inhibitors. Gynecol Oncol 116: 424-429.

Packenham JP, du Manoir S, Schrock E, Risinger JI, Dixon D, Denz DN, Evans JA, Berchuck A, Barrett JC, Devereux TR, et al. 1997. Analysis of genetic alterations in uterine leiomyomas and leiomyosarcomas by comparative genomic hybridization. Mol Carcinog 19: 273-279. 
Pérot G, Croce S, Ribeiro A, Lagarde P, Vélasco V, Neuville A, Coindre JM, Stoeckle E, Floquet A, MacGrogan G, et al. 2012. MED12 alterations in both human benign and malignant uterine soft tissue tumors. PLoS One 7: e40015.

Pfeifer W, Sokolenko AP, Potapova ON, Bessonov AA, Ivantsov AO, Laptiev SA, Zaitseva OA, Yatsuk OS, Matsko DE, Semiglazova TY, et al. 2014. Breast cancer sensitivity to neoadjuvant therapy in BRCA1 and CHEK2 mutation carriers and non-carriers. Breast Cancer Res Treat 148: 675-683.

Quade BJ, Weremowicz S, Neskey DM, Vanni R, Ladd C, Dal Cin P, Morton CC. 2003. Fusion transcripts involving HMGA2 are not a common molecular mechanism in uterine leiomyomata with rearrangements in 12q15. Cancer Res 63: 1351-1358.

Rausch T, Zichner T, Schlattl A, Stütz AM, Benes V, Korbel JO. 2012. DELLY: structural variant discovery by integrated paired-end and split-read analysis. Bioinformatics 28: i333-i339.

Robertson G, Schein J, Chiu R, Corbett R, Field M, Jackman SD, Mungall K, Lee S, Okada HM, Qian JQ, et al. 2010. De novo assembly and analysis of RNA-seq data. Nat Methods 7: 909-912.

Saunders CT, Wong WSW, Swamy S, Becq J, Murray LJ, Cheetham RK. 2012. Strelka: accurate somatic smallvariant calling from sequenced tumor-normal sample pairs. Bioinformatics 28: 1811-1817.

Sheffield BS, Fulton R, Kalloger SE, Milne K, Geller G, Jones M, Jacquemont C, Zachara S, Zhao E, Pleasance E, et al. 2016. Investigation of PD-L1 biomarker testing methods for PD-1 axis inhibition in non-squamous non-small cell lung cancer. J Histochem Cytochem 64: 587-600.

Simpson JT, Wong K, Jackman SD, Schein JE, Jones SJM, Birol I. 2009. ABySS: a parallel assembler for short read sequence data. Genome Research 19: 1117-1123.

Smith KL, Isaacs C. 2011. BRCA mutation testing in determining breast cancer therapy. Cancer J 17: 492-499

Suspitsin EN, Yanus GA, Sokolenko AP, Yatsuk OS, Zaitseva OA, Bessonov AA, Ivantsov AO, Heinstein VA, Klimashevskiy VF, Togo AV, et al. 2014. Development of breast tumors in CHEK2, NBN/NBS1 and BLM mutation carriers does not commonly involve somatic inactivation of the wild-type allele. Med Oncol 31: 828.

Suwaki N, Klare K, Tarsounas M. 2011. RAD51 paralogs: roles in DNA damage signalling, recombinational repair and tumorigenesis. Semin Cell Dev Biol 22: 898-905.

Telli ML, Jensen KC, Vinayak S, Kurian AW, Lipson JA, Flaherty PJ, Timms K, Abkevich V, Schackmann EA, Wapnir IL, et al. 2015. Phase II study of gemcitabine, carboplatin, and iniparib as neoadjuvant therapy for triple-negative and BRCA1/2 mutation-associated breast cancer with assessment of a tumor-based measure of genomic instability: PrECOG 0105. J Clin Oncol 33: 1895-1901.

The Cancer Genome Atlas (TCGA) Research Network, http://cancergenome.nih.gov/.

Thompson LH. 2012. Recognition, signaling, and repair of DNA double-strand breaks produced by ionizing radiation in mammalian cells: the molecular choreography. Mutat Res 751: 158-246.

Timms KM, Abkevich V, Hughes E, Neff C, Reid J, Morris B, Kalva S, Potter J, Tran TV, Chen J, et al. 2014. Association of BRCA1/2 defects with genomic scores predictive of DNA damage repair deficiency among breast cancer subtypes. Breast Cancer Res 16: 475.

Toro JR, Travis LB, Wu HJ, Zhu K, Fletcher CDM, Devesa SS. 2006. Incidence patterns of soft tissue sarcomas, regardless of primary site, in the surveillance, epidemiology and end results program, 1978-2001: an analysis of 26,758 cases. Int J Cancer 119: 2922-2930.

Ul-Hassan A, Sisley K, Hughes D, Hammond DW, Robinson MH, Reed MWR. 2009. Common genetic changes in leiomyosarcoma and gastrointestinal stromal tumour: implication for ataxia telangiectasia mutated involvement. Int J Exp Pathol 90: 549-557.

Vahteristo P, Tamminen A, Karvinen P, Eerola H, Eklund C, Aaltonen LA, Blomqvist C, Aittomäki K, Nevanlinna H. 2001. p53, CHK2, and CHK1 genes in Finnish families with Li-Fraumeni syndrome: further evidence of CHK2 in inherited cancer predisposition. Cancer Res 61: 5718-5722.

Ward A, Khanna KK, Wiegmans AP. 2015. Targeting homologous recombination, new pre-clinical and clinical therapeutic combinations inhibiting RAD51. Cancer Treat Rev 41: 35-45.

Weischer M, Bojesen SE, Ellervik C, Tybjaerg-Hansen A, Nordestgaard BG. 2008. CHEK2*1100delC genotyping for clinical assessment of breast cancer risk: meta-analyses of 26,000 patient cases and 27,000 controls. $J$ Clin Oncol 26: 542-548.

Weischer M, Nordestgaard BG, Pharoah P, Bolla MK, Nevanlinna H, Van't Veer LJ, García-Closas M, Hopper JL, Hall P, Andrulis IL, et al. 2012. CHEK2*1100delC heterozygosity in women with breast cancer associated with early death, breast cancer-specific death, and increased risk of a second breast cancer. $\mathrm{J}$ Clin Oncol 30: 4308-4316.

Xu X, Tsvetkov LM, Stern DF. 2002. Chk2 activation and phosphorylation-dependent oligomerization. Mol Cell Biol 22: 4419-4432.

Yang C-Y, Liau J-Y, Huang W-J, Chang Y-T, Chang M-C, Lee J-C, Tsai J-H, Su Y-N, Hung C-C, Jeng Y-M. 2015. Targeted next-generation sequencing of cancer genes identified frequent TP53 and ATRX mutations in leiomyosarcoma. Am J Transl Res 7: 2072-2081.

Zannini L, Delia D, Buscemi G. 2014. CHK2 kinase in the DNA damage response and beyond. J Mol Cell Biol 6: 442-457. 


\section{COLD SPRING HARBOR Molecular Case Studies}

\section{Genomic profiling of pelvic genital type leiomyosarcoma in a woman with a germline CHEK2:C.1100delC mutation and a concomitant diagnosis of metastatic invasive ductal breast carcinoma}

My Linh Thibodeau, Caralyn Reisle, Eric Zhao, et al.

Cold Spring Harb Mol Case Stud 2017, 3: a001628 originally published online May 16, 2017 Access the most recent version at doi: $10.1101 /$ mcs.a001628

Supplementary Material

References

License

Email Alerting Service
http://molecularcasestudies.cshlp.org/content/suppl/2017/05/16/mcs.a001628.D C1

This article cites 70 articles, 16 of which can be accessed free at: http://molecularcasestudies.cshlp.org/content/3/5/a001628.full.html\#ref-list-1

This article is distributed under the terms of the Creative Commons Attribution-NonCommercial License, which permits reuse and redistribution, except for commercial purposes, provided that the original author and source are credited.

Receive free email alerts when new articles cite this article - sign up in the box at the top right corner of the article or click here. 